\title{
Las estrategias de lectura y su incidencia en la comprensión lectora de estudiantes de una universidad pública del noroeste de México
}

\author{
Reading Strategies and Their Impact on the Reading Comprehension of Students \\ at a Public University in Northwestern Mexico
}

\section{Estratégias de leitura e seu impacto na compreensão de leitura de estudantes de uma universidade pública no noroeste do México}

\author{
Aidee Espinosa Pulido \\ Universidad Autónoma de Baja California, México \\ aidee.espinosa@uabc.edu.mx \\ https://orcid.org/0000-0003-3976-9514
}

\section{Resumen}

Este trabajo presenta los resultados de una investigación que tuvo como objetivo indagar si es posible mejorar la comprensión lectora de los estudiantes de primer semestre de una institución pública del noroeste de México mediante estrategias de lectura. Se utilizó una metodología cuantitativa de carácter descriptiva. El tipo de diseño de investigación fue preexperimental con un solo grupo pretest y postest para la comprobación de la hipótesis planteada. El procedimiento fue evaluar con un pretest la comprensión lectora de los participantes para conocer el nivel en que se encontraban antes de la instrucción. Se continuó con la implementación de un taller de diez horas para instruir el uso de estrategias de lectura. Finalmente, se evaluó con un postest la comprensión lectora para comparar los resultados de ambos test y contar con elementos para determinar la efectividad o no de la intervención. Los resultados demostraron que después de la instrucción $38 \%$ de los participantes avanzó de nivel y 50 \% de los casos, aunque no logró cambiar de nivel, 


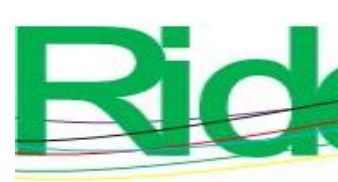

Revista Iberoamericana para la
Investigación y el Desarrollo Educativo
ISSN $2007-7467$

mejoró su comprensión lectora al obtener mayor puntaje. Por lo anterior, se recomienda la instrucción en el uso de estrategias y con ello favorecer la formación de lectores competentes.

Palabras clave: comprensión lectora, estrategias de lectura, formación universitaria.

\section{Abstract}

This work presents the results of a research that aimed to investigate whether it is possible to improve the reading comprehension of the students from the first semester of a public institution in the northwest of Mexico through reading strategies. A descriptive quantitative methodology was used. The type of research design was pre-experimental with a single pre-test and post-test group to check the hypothesis. The procedure was to assess the reading comprehension of the participants with a pretest to know the level they were in before the instruction. An intervention plan was continued: a ten-hour workshop was implemented to instruct the use of reading strategies. Finally, reading comprehension was evaluated with a post-test to compare the results of both tests and to have elements to determine the effectiveness or not of the intervention. The results showed that after the instruction on the use of strategies $38 \%$ of the study participants advanced in level and $50 \%$ of the cases, although they did not manage to change level, improved their reading comprehension by obtaining a higher score. Therefore, instruction in the use of strategies is recommended, and with this, the training of competent readers is encouraged.

Keywords: reading comprehension, reading strategies, university training.

\section{Resumo}

Este trabalho apresenta os resultados de uma pesquisa que teve como objetivo investigar se é possível melhorar a compreensão de leitura dos alunos do primeiro semestre de uma instituição pública no noroeste do México por meio de estratégias de leitura. Foi utilizada uma metodologia quantitativa descritiva. $\mathrm{O}$ tipo de desenho da pesquisa foi pré-experimental, com um único grupo de pré-teste e pós-teste para verificar a hipótese. O procedimento foi avaliar com um pré-teste a compreensão de leitura dos participantes para saber o nível em que estavam antes da instrução. A implementação de um workshop de dez horas continuou a instruir o uso de estratégias de leitura. Finalmente, a compreensão da leitura foi avaliada com um pós-teste para comparar os resultados de ambos os testes e ter elementos para determinar a eficácia ou não da intervenção. Os resultados mostraram que, após a instrução, 38\% dos participantes avançaram em nível e 50\% 


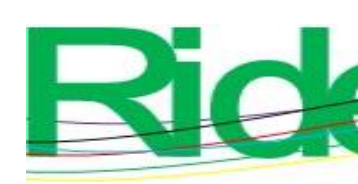

Revista Iberoamericana para la Investigación y el Desarrollo Educativo ISSN 2007 - 7467

dos casos, embora não tenham conseguido alterar o nível, sua compreensão de leitura melhorou com a obtenção de uma pontuação mais alta. Portanto, recomenda-se instruções sobre o uso de estratégias e, com isso, o treinamento de leitores competentes é incentivado.

Palavras-chave: compreensão de leitura, estratégias de leitura, ensino universitário.

Fecha Recepción: Enero 2020

Fecha Aceptación: Julio 2020

\section{Introducción}

En la formación de universitarios se asume que estos cuentan con un buen nivel de comprensión lectora, dado que es una de las habilidades que presuntamente debieron haber perfeccionado en los grados académicos anteriores. Sin embargo, en mi experiencia como docente observo fallas de comprensión en lecturas asignadas a los estudiantes. A partir de ello una pregunta se ha ido abriendo camino: ¿qué estrategias de apoyo de comprensión lectora utilizan los dicentes de este nivel?

La Organización de las Naciones Unidas para la Educación, la Ciencia y la Cultura [Unesco] (2000, citada en Gutiérrez y Montes de Oca, 2016) plantea que saber leer constituye una capacidad necesaria en sí misma, y es la base de otras aptitudes vitales: "Los libros y el acto de leer constituyen los pilares de la educación y la difusión del conocimiento, la democratización de la cultura y la superación individual y colectiva de los seres humanos" (p. 4). Sin duda la lectura es una tarea pendiente en la agenda de instituciones importantes en el ámbito mundial.

El comité de expertos de la Organización para la Cooperación y el Desarrollo Económicos [OCDE] (2007) define a la comprensión lectora como "la capacidad de un individuo para comprender, utilizar y reflexionar sobre textos escritos, con el propósito de alcanzar sus objetivos personales, desarrollar su conocimiento y capacidades, y participar en la sociedad" (p. 7). Por cuestiones como estas, señaladas por dicha organización, es que el lector debe tener claro con qué intención lee para que su lectura sea más efectiva.

En México se han desarrollado varios trabajos en relación con la comprensión lectora. Entre ellos el de Vidal y Manríquez (2016), quienes señalan la deficiencia en esta competencia por parte de quienes ingresan al nivel de formación profesional. Por otro lado, Gaeta (2015) destaca como parte de sus resultados la relevancia de los factores cognitivos y motivacionales que se involucran en la comprensión de textos académicos en el contexto universitario mexicano. Además, confirma que los alumnos usan las estrategias para autorregular el aprendizaje. Para 


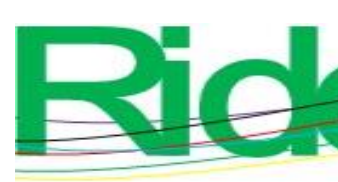

Revista Iberoamericana para la Investigación y el Desarrollo Educativo ISSN $2007-7467$

lograr la comprensión se debe contar con conocimientos previos y adoptar metas intrínsecas que promuevan la habilidad y el aprendizaje.

Cáceres, Pérez y Zúñiga (2018) concluyen que la lectura es una actividad cognitiva de gran importancia y complejidad utilizada normalmente para la adquisición de conocimientos. Dicha actividad se convierte en la herramienta principal de aprendizaje para los estudiantes, puesto que la mayoría de las actividades académicas se basan en la lectura. Por tanto, el éxito académico en todos los niveles escolares y principalmente en el ámbito de la educación superior dependerá, en gran medida, de las estrategias y recursos que posee un buen lector.

Si analizamos el contexto anterior a la educación universitaria, es decir, la educación media superior, se advierte que el problema de la comprensión lectora proviene de deficiencias no superadas en años de formación anteriores. Una evidencia de ello son los resultados obtenidos en el Programa Internacional para la Evaluación de Estudiantes (PISA, por sus siglas en inglés), el cual es un estudio llevado a cabo por la OCDE que evalúa el rendimiento de estudiantes de 15 años en las áreas como matemáticas, ciencias y lectura. De esta última área es la información que es relevante para lo aquí abordado: México mostró un bajo desempeño de lectura, de los 65 países participantes, se posicionó en el número 52 (OCDE, 2016).

Se espera que los estudiantes universitarios cuenten con la habilidad de la comprensión lectora, y así, puedan analizar, sintetizar, evaluar y criticar información de diversas fuentes, lo que les brindará herramientas suficientes para su desarrollo académico. Los docentes, en la formación profesional, señalan lecturas cada vez más complejas con la intención fortalecer los aprendizajes de los alumnos, mas es una tarea ardua cuando se observa que los alumnos no cuentan con esta habilidad (Vidal y Manríquez, 2016). En una evaluación informal de la comprensión lectora, a partir de los resultados que obtuvimos, destaca que uno de los problemas de los alumnos universitarios es su dificultad para seleccionar la información importante de los textos, lo cual está relacionado con el hecho de que no utilizan estrategias efectivas para extraer las ideas principales (Guevara, Guerra, Delgado y Flores, 2014).

Osorio, Mendoza y Ballesteros (2018) afirman que la lectura es un medio de acceso a la información y uno de los caminos para contribuir a la generación de conocimiento. Actualmente se ha demostrado que si el individuo está capacitado y posee la competencia lectora, por consiguiente tendrá mejores condiciones para desarrollarse en la sociedad actual, a la que se le ha denominado de la información y el conocimiento, la cual, como bien sabemos, se caracteriza por experimentar transformaciones aceleradas a través de la generación, uso y difusión de la 


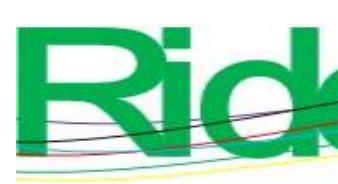

Revista Iberoamericana para la Investigación y el Desarrollo Educativo ISSN $2007-7467$

información, materia prima y esencial del conocimiento humano. Y es en estos escenarios que la lectura se constituye como la llave de acceso a una sociedad que conoce, comprende, consolida, analiza, sintetiza, aplica, construye y reconstruye los saberes de la humanidad.

Cassany (2006) señala que leer es comprender y, concretamente, es elaborar significados que no se mencionan explícitamente en el texto, cuestión a la que se le denomina inferencia. Los significados los construye el lector mentalmente a partir de estímulos del texto y de su conocimiento previo. La comprensión lectora tiene que ver con una participación activa del lector, poniendo en juego lo que conoce de las palabras y lo que estas dicen entre líneas, aunado a lo que conoce del tema para poder dar y expandir el significado que el texto ofrece.

Leer es un proceso de interacción entre el lector y el texto para obtener la información pertinente según los objetivos que guían su lectura (Solé, 2011). Este proceso requiere de un lector activo que procesa y examina un texto. Por lo anterior, para que se dé la comprensión lectora, quien lee debe tener un claro interés y objetivo delimitado para poder extraer del texto la información que este le brinda. Felipe y Barrios (2017) afirman que la lectura es una competencia clave, cuya importancia reside en la capacidad instrumental indispensable para acceder a saberes y desarrollar capacidades propias de las enseñanzas universitarias.

Mientras que Valles (2005) señala que en la comprensión lectora intervienen procesos psicológicos básicos como:

1) Atención selectiva: donde el lector debe focalizar su atención en el texto según su objeto de lectura.

2) Realizar un análisis secuencial: proceso mental de análisis que consiste en una lectura continuada, palabra tras palabra, conectando el significado de cada una de ellas.

3) Síntesis: proceso donde el lector recapitula, resume y da significado a las unidades lingüísticas, para que las palabras cobren unidad coherente y con significado, es decir, la comprensión del texto.

4) Memoria: el proceso de lectura y su comprensión se logra mediante rutinas de almacenamiento.

Por lo que se puede observar que la lectura comprensiva requiere de un proceso más intencionado de lo que normalmente los estudiantes atienden.

Como hemos visto, la comprensión lectora es un proceso activo donde hay interacción entre el texto y el lector. Pero dicha interacción no es monocromática, sino que implica distintos 


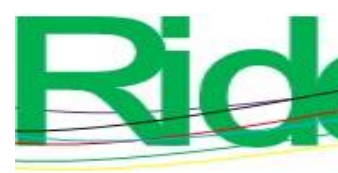

Revista Iberoamericana para la
Investigación y el Desarrollo Educativo
ISSN $2007-7467$

matices, esto es, el lector establece distintos grados de entendimiento respecto al texto. Así, Smith (1989, citado por Gordillo y Flores, 2009) habla de tres niveles de comprensión:

- $\quad$ Nivel de comprensión literal. En este primer nivel, el lector es capaz de reconocer las palabras y las frases y capta la estructura del texto. La intervención intelectual y cognoscitiva del lector no es muy activa.

- $\quad$ Nivel de comprensión inferencial. Segundo nivel donde el lector relaciona y asocia el significado de las palabras, lo que le permite leer entre líneas, presuponer y deducir lo implícito, es capaz de relacionar lo leído con los conocimientos previos y elaborar conclusiones.

- $\quad$ Nivel de comprensión crítico. En este último nivel, el lector es capaz de emitir un juicio aceptando o rechazando con argumentos la información del texto leído.

Saber leer y alcanzar un grado profundo de comprensión no es una tarea sencilla; se puede lograr cuando el lector esté dispuesto a participar activamente en la interacción con el texto (Parodi, Peronard e Ibáñez, 2010). En la instrucción se debe motivar a los estudiantes con lecturas que les sean atrayentes e interesantes, así como asegurarnos que logren detectar lo que el texto aporta no solo para sus conocimientos, sino para su formación en general. Asimismo, en el acto de leer es importante saber identificar y determinar previamente la estructura del texto, con ello al lector se le facilitará entender la información contenida en el documento (Van Dijk \& Kintsch, 1983). Cuando los estudiantes reconozcan las diferentes estructuras textuales, la argumentativa, narrativa o descriptiva, entre otras, contarán con elementos para interpretar y organizar la información textual durante el acto de leer.

Por último, cabe señalar que en este trabajo nos centramos en textos expositivos. Corbacho (2006) los define como documentos que están asociados al análisis y la síntesis de ideas y representaciones conceptuales. Este tipo de textos clasifican, explican y definen conceptos. Se trata de textos que están ligados a las tareas escolares. 


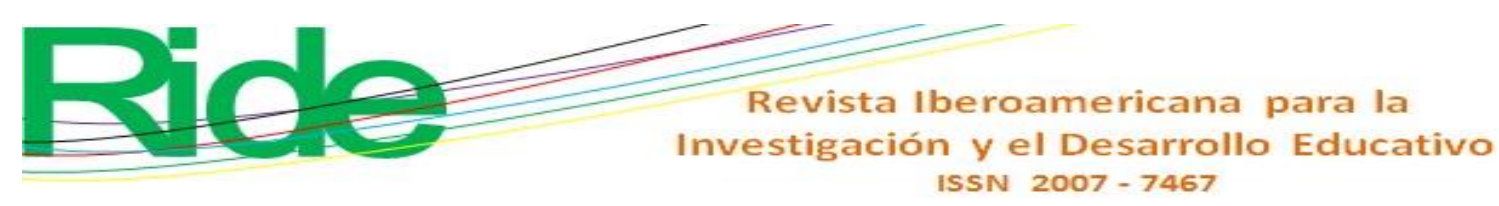

\section{Metodología}

En esta investigación se utilizó un enfoque cuantitativo porque los datos se obtuvieron con valores exactos, lo cual asegura la precisión cuantificable del problema. Este enfoque suele utilizarse cuando se quieren estimar las magnitudes u ocurrencia de los fenómenos y probar hipótesis. Esto mediante el planteamiento de un problema de estudio, acotado sobre el fenómeno de interés, en un contexto concreto, en este caso la competencia lectora en universitarios (Hernández y Mendoza, 2018).

El diseño fue documental, pues se pretendió ampliar la información de las variables mediante la recopilación de datos de diversas fuentes de investigación y así aportar nuevos conocimientos acerca de las estrategias de lectura, todo con la intención de potenciar la comprensión lectora de los universitarios (Arias, 2012). El tipo de investigación utilizado fue el descriptivo, dado que se aborda el problema detallando sus características y describiendo sus variables. Además, se interpreta y especifica la información de las encuestas para la elaboración de conclusiones.

Se inició con un diagnóstico, instrumento de elaboración propia, mediante un cuestionario construido con preguntas explícitas para buscar respuestas sobre el uso de estrategias de lectura. Lo anterior, al ser la primera necesidad indagar si los participantes de estudio empleaban estrategias de lectura como apoyo para comprender los textos que les son asignados por sus docentes en la universidad. En la tabla 1 se pueden observar los resultados. 


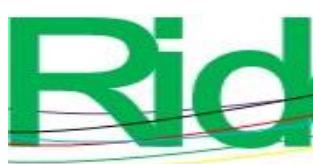

Revista Iberoamericana para la Investigación y el Desarrollo Educativo ISSN 2007 - 7467

Tabla 1. Resultados de diagnóstico: encuesta basada en el Cuestionario sobre uso de Estrategias de Lectura. Presentada en porcentajes. Total de la muestra: 33 estudiantes

\begin{tabular}{|c|c|c|c|c|c|}
\hline Pregunta & Nunca & $\begin{array}{c}\text { Casi } \\
\text { nunca }\end{array}$ & $\begin{array}{c}\text { Algunas } \\
\text { veces }\end{array}$ & Frecuentemente & Siempre \\
\hline $\begin{array}{l}\text { 1) ¿Realizas resúmenes o } \\
\text { síntesis para apoyar la } \\
\text { comprensión de los } \\
\text { textos que lees? }\end{array}$ & $10 \%$ & $30 \%$ & $44 \%$ & $10 \%$ & $6 \%$ \\
\hline $\begin{array}{l}\text { 2) ¿Elaboras mapas } \\
\text { conceptuales para } \\
\text { mejorar la comprensión } \\
\text { de tus lecturas? }\end{array}$ & $17 \%$ & $47 \%$ & $27 \%$ & $8 \%$ & $1 \%$ \\
\hline $\begin{array}{l}\text { 3) ¿Utilizas la técnica del } \\
\text { subrayado para apoyar } \\
\text { la comprensión lectora? }\end{array}$ & $5 \%$ & $7 \%$ & $27 \%$ & $37 \%$ & $24 \%$ \\
\hline $\begin{array}{lrr}\text { 4) ¿Identificas las } & \text { ideas } \\
\text { principales } & & y \\
\text { secundarias de } & \text { los } \\
\text { textos que lees? } & \end{array}$ & $6 \%$ & $7 \%$ & $27 \%$ & $36 \%$ & $24 \%$ \\
\hline $\begin{array}{l}\text { 5) ¿Buscas la definición de } \\
\text { palabras desconocidas } \\
\text { en los textos que lees? }\end{array}$ & $5 \%$ & $9 \%$ & $21 \%$ & $30 \%$ & $35 \%$ \\
\hline $\begin{array}{l}\text { 6) ¿Planteas una hipótesis } \\
\text { en relación con lo que } \\
\text { leerás para orientar tu } \\
\text { lectura? }\end{array}$ & $8 \%$ & $31 \%$ & $37 \%$ & $20 \%$ & $4 \%$ \\
\hline $\begin{array}{l}\text { 7) ¿Reflexionas sobre lo } \\
\text { que lees? }\end{array}$ & $2 \%$ & $8 \%$ & $36 \%$ & $33 \%$ & $21 \%$ \\
\hline
\end{tabular}

Fuente: Elaboración propia

El diagnóstico demuestra que el uso de estrategias es variable, pero, en general, no es una constante en el apoyo para la comprensión lectora. Con esta información se formuló el planteamiento del problema y se seleccionó una metodología con la que se pudiera contrarrestar 

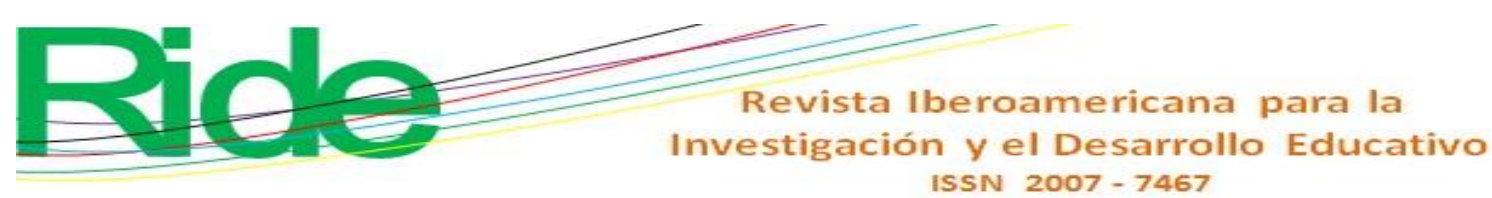

esta cuestión, puesto que, hay que recordar, el interés de la investigación tuvo como base ayudar a los estudiantes para mejorar su comprensión lectora.

Dicho planteamiento quedó de la siguiente forma: ¿la instrucción en el uso de estrategias tiene incidencia en la comprensión lectora de los estudiantes de primer semestre de la Facultad de Pedagogía e Innovación Educativa de la Universidad Autónoma de Baja California inscritos en tronco común en el ciclo 2019-1?

\section{Hipótesis}

La instrucción sobre el uso de estrategias de lectura tendrá incidencia en la comprensión lectora de los estudiantes de primer semestre participantes.

- Variable independiente: Estrategias de lectura.

- Variable dependiente: Comprensión lectora.

\section{Objetivo general}

Investigar si la instrucción sobre el uso de estrategias de lectura incide de forma positiva en la comprensión lectora.

\section{Objetivos específicos}

1) Diagnosticar el uso de estrategias de lectura por los estudiantes de primer semestre de la Facultad de Pedagogía.

2) Establecer el nivel de comprensión lectora de los participantes.

3) Definir un plan de intervención para instruir a los participantes en el uso de estrategias de lectura.

4) Determinar si la instrucción sobre el uso de estrategias de lectura favorece la comprensión lectora.

Se manejó un diseño preexperimental con un solo grupo pretest y postest para la comprobación de la hipótesis planteada en el estudio. La ejecución del diseño con un solo grupo pretest-postest implica, primero, una medición previa a la aplicación de la variable dependiente a ser estudiada $\mathrm{O}_{1}$ (pretest); segundo, la introducción o aplicación del tratamiento de la variable independiente o experimental $(\mathrm{X})$, y tercero, una nueva medición de la variable dependiente $\mathrm{O}_{2}$ (postest), lo cual es diagramado como $\mathrm{O}_{1} \mathrm{X} \mathrm{O}_{2}$ (Espinosa, 2016). 

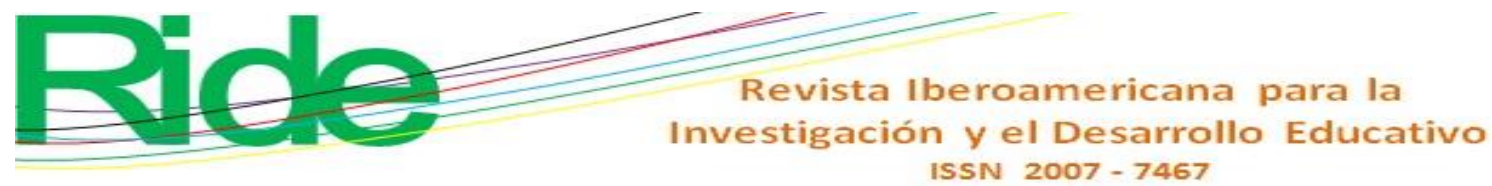

Buendía (2000) señala que el efecto del tratamiento del diseño preexperimental con un solo grupo se comprueba cuando se comparan los resultados $\mathrm{O}_{1}$ vs. $\mathrm{O}_{2}$. En este caso, se contrastaron los resultados del postest con los de pretest. Este tipo de diseño requiere que la aplicación de ambas evaluaciones sea lo más parecida posible para evitar que los resultados varíen. De acuerdo con esta estructura, se delineó la siguiente tabla para aclarar el diseño.

Tabla 2. Tipo de diseño. Preexperimental con un solo grupo

\begin{tabular}{|c|c|c|}
\hline $\mathrm{O}_{1}$ & $\mathrm{X}$ & $\mathrm{O}_{2}$ \\
Pretest & Tratamiento & Postest \\
Cuestionario para & Plan de intervención. Taller & Cuestionario para \\
determinar el nivel de & sobre uso de estrategias de & determinar el nivel de \\
comprensión lectora & lectura & comprensión lectora \\
\hline
\end{tabular}

Fuente: Elaboración propia

En este diseño la $\mathrm{O}_{1}$ representa al grupo de los 33 participantes de estudio previo a la aplicación de la variable, que, como ya se ha indicado en esta investigación, se refiere al plan de intervención representado por la $X$, y la $\mathrm{O}_{2}$ representa al mismo grupo posterior a la intervención. El plan de intervención se implementó mediante un taller de cinco sesiones con duración de dos horas cada una. Cada sesión contempló la instrucción sobre el uso de una estrategia de lectura.

- $\quad 1$ a $^{\text {a }}$ sesión. Estrategia de lectura: subrayado e identificación de ideas esenciales.

- $\quad 2$ a $^{\text {a }}$ sesión. Estrategia de lectura: despejar lagunas de comprensión. La búsqueda de palabras desconocidas.

- $\quad 3 .^{a}$ sesión . Estrategia de lectura: organizadores gráficos: mapas conceptuales.

- $\quad$ 4. ${ }^{\mathrm{a}}$ sesión . Estrategia de lectura: la hipótesis como estrategia de lectura.

- $\quad 5$ a $^{\mathrm{a}}$ sesión . Estrategia de lectura: reflexión sobre lo leído.

La medición pretest y postest evaluó la comprensión lectora. Para ello, se diseñaron dos cuestionarios, uno para cada una de las mediciones. Estos fueron construidos con preguntas de opción múltiple para comprobar la comprensión del texto. Se eligió este instrumento porque puede aplicarse a un gran número de alumnos, además de que se reduce la selección de respuesta al azar (Pérez, 2005). En la elaboración de los instrumentos se hizo una adaptación del cuestionario de Gordillo y Flores (2009), por lo que se contemplaron dos textos expositivos con 20 párrafos, de donde se generaron preguntas para determinar el nivel de comprensión lectora: de 

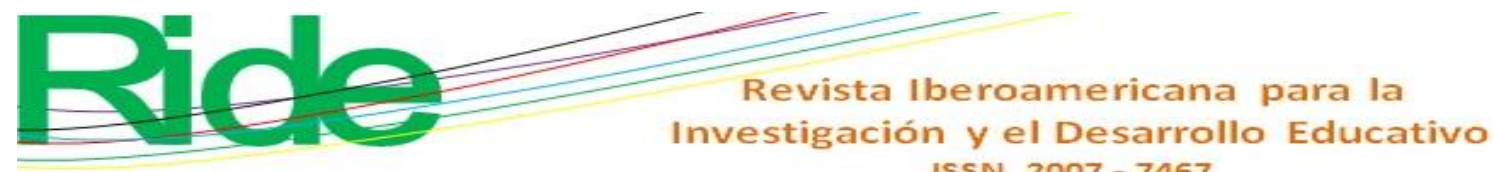

En cuanto a las técnicas e instrumentos de recolección de datos, se seleccionó una investigación de campo en la que se empleó la técnica de encuesta-escrita, para lo que se diseñaron como instrumentos cuestionarios construidos con ítems acordes a la información que se pretendía conocer de los participantes del estudio (Arias, 2012).

\section{Resultados}

Los resultados de la investigación se obtuvieron al contrastar los datos que se obtuvieron de las pruebas pretest y postest (ver tabla 4). 
Tabla 4. Resultados de la evaluación de niveles de comprensión lectora: pretest/postest

\begin{tabular}{|c|c|c|c|c|c|c|}
\hline \multirow[b]{2}{*}{ Participantes } & \multicolumn{2}{|c|}{ Resultados pretest } & \multicolumn{2}{|c|}{ Resultados postest } & \multirow{2}{*}{$\begin{array}{c}\text { Avance de } \\
\text { nivel }\end{array}$} & \multirow{2}{*}{$\begin{array}{l}\text { Mayor } \\
\text { puntaje }\end{array}$} \\
\hline & Puntaje & $\begin{array}{l}\text { Nivel de } \\
\text { lectura }\end{array}$ & Puntaje & $\begin{array}{c}\text { Nivel de } \\
\text { lectura }\end{array}$ & & \\
\hline 1 & 20 & Inferencial & 30 & Inferencial & $=$ & 10 \\
\hline 2 & 20 & Inferencial & 35 & Crítico & 1 & 15 \\
\hline 3 & 20 & Inferencial & 30 & Inferencial & $=$ & 10 \\
\hline 4 & 30 & Inferencial & 40 & Crítico & 1 & 10 \\
\hline 5 & 15 & Literal & 25 & Inferencial & 1 & 10 \\
\hline 6 & 30 & Inferencial & 35 & Crítico & 1 & 5 \\
\hline 7 & 35 & Crítico & 35 & Crítico & $=$ & 0 \\
\hline 8 & 20 & Inferencial & 35 & Crítico & 1 & 15 \\
\hline 9 & 35 & Crítico & 35 & Crítico & $=$ & 0 \\
\hline 10 & 40 & Crítico & 40 & Crítico & $=$ & 0 \\
\hline 11 & 20 & Inferencial & 30 & Inferencial & $=$ & 10 \\
\hline 12 & 20 & Inferencial & 25 & Inferencial & $=$ & 5 \\
\hline 13 & 10 & Literal & 35 & Crítico & 2 & 20 \\
\hline 14 & 25 & Inferencial & 30 & Inferencial & $=$ & 5 \\
\hline 15 & 25 & Inferencial & 40 & Crítico & 1 & 15 \\
\hline 16 & 35 & Crítico & 40 & Crítico & $=$ & 5 \\
\hline 17 & 20 & Inferencial & 30 & Inferencial & $=$ & 10 \\
\hline 18 & 20 & Inferencial & 25 & Inferencial & $=$ & 5 \\
\hline 19 & 15 & Literal & 30 & Inferencial & 1 & 15 \\
\hline 20 & 25 & Inferencial & 35 & Crítico & 1 & 10 \\
\hline 21 & 20 & Inferencial & 30 & Inferencial & $=$ & 10 \\
\hline 22 & 35 & Crítico & 40 & Crítico & $=$ & 5 \\
\hline 23 & 30 & Inferencial & 35 & Crítico & 1 & 5 \\
\hline 24 & 20 & Inferencial & 25 & Inferencial & $=$ & 5 \\
\hline 25 & 35 & Crítico & 35 & Crítico & $=$ & 0 \\
\hline 26 & 25 & Inferencial & 30 & Inferencial & $=$ & 5 \\
\hline 27 & 40 & Crítico & 40 & Crítico & $=$ & 0 \\
\hline
\end{tabular}




\begin{tabular}{|c|c|l|c|l|l|c|}
\hline 28 & 30 & Inferencial & 35 & Crítico & 1 & 5 \\
\hline 29 & 35 & Crítico & 40 & Crítico & $=$ & 5 \\
\hline 30 & 15 & Literal & 35 & Crítico & 2 & 20 \\
\hline 31 & 35 & Crítico & 40 & Crítico & $=$ & 5 \\
\hline 32 & 25 & Inferencial & 35 & Crítico & 1 & 10 \\
\hline 33 & 25 & Inferencial & 35 & Crítico & 1 & 10 \\
\hline
\end{tabular}

Fuente: Elaboración propia

Para el análisis de los resultados, se construyó la tabla de distribución de frecuencias para medir las variables: tabla 5 para los de pretest y tabla 6 para los de postest.

Tabla 5. Distribución de frecuencias para medición de variables de resultados pretest

\begin{tabular}{|c|c|c|c|c|c|c|}
\hline 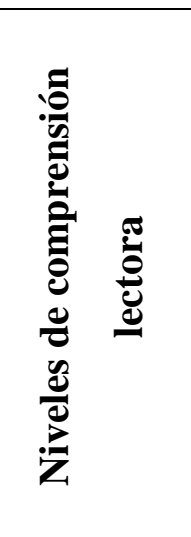 & 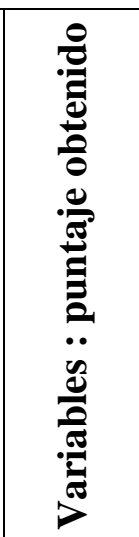 & 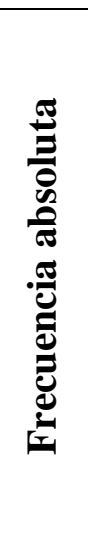 & 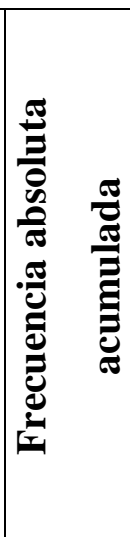 & 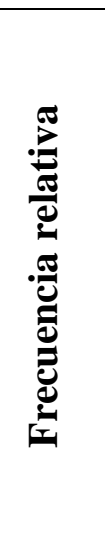 & 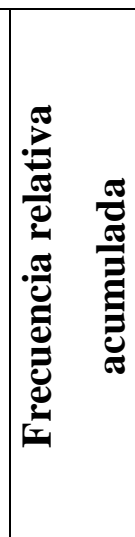 & 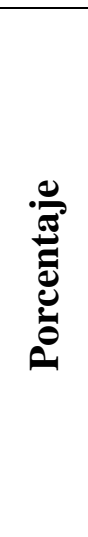 \\
\hline NCL & $x i$ & fi & $\mathrm{Fi}$ & hi & $\mathrm{Hi}$ & $\%$ \\
\hline Literal & 10 & 10 & 10 & 0.30 & 0.30 & 30 \\
\hline Literal & 15 & 5 & 15 & 0.15 & 0.45 & 15 \\
\hline Inferencial & 20 & 5 & 20 & 0.15 & 0.61 & 15 \\
\hline Inferencial & 25 & 5 & 25 & 0.15 & 0.76 & 15 \\
\hline Inferencial & 30 & 5 & 30 & 0.15 & 0.91 & 15 \\
\hline Crítico & 35 & 3 & 33 & 0.09 & 1.00 & 9 \\
\hline Crítico & 40 & 0 & 33 & 0.00 & 1.00 & 0 \\
\hline & Totales & 33 & & 1.00 & & 100 \\
\hline
\end{tabular}

Fuente: Elaboración propia 


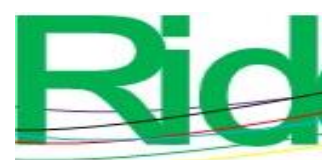

Revista Iberoamericana para la Investigación y el Desarrollo Educativo ISSN 2007 - 7467

Tabla 6. Distribución de frecuencias para medición de variables de resultados postest

\begin{tabular}{|c|c|c|c|c|c|c|}
\hline 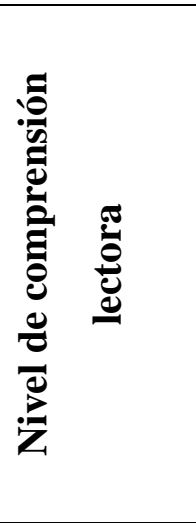 & 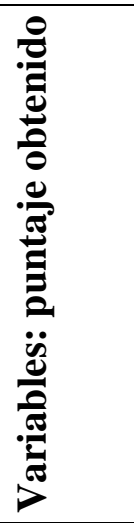 & 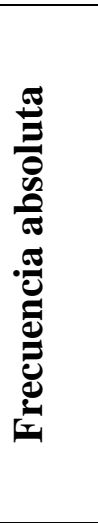 & 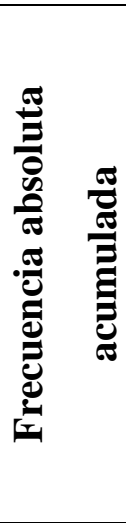 & 胥 & تِّ & 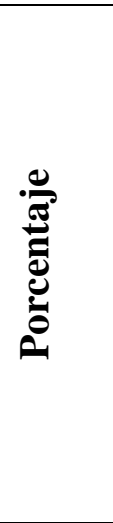 \\
\hline NCL & $x i$ & fi & $\mathrm{Fi}$ & hi & $\mathrm{Hi}$ & $\%$ \\
\hline Inferencial & 25 & 4 & 4 & 0.12 & 0.12 & 12 \\
\hline Inferencial & 30 & 8 & 12 & 0.24 & 0.36 & 24 \\
\hline Crítico & 35 & 13 & 25 & 0.39 & 0.76 & 39 \\
\hline \multirow[t]{2}{*}{ Crítico } & 40 & 8 & 33 & 0.24 & 1.00 & 24 \\
\hline & Totales & 33 & & 1.00 & & 100 \\
\hline
\end{tabular}

Fuente: Elaboración propia

Se graficaron los resultados de cada test en porcentajes para clarificar la información (ver figuras 1 y 2).

Figura 1. Resultados pretest

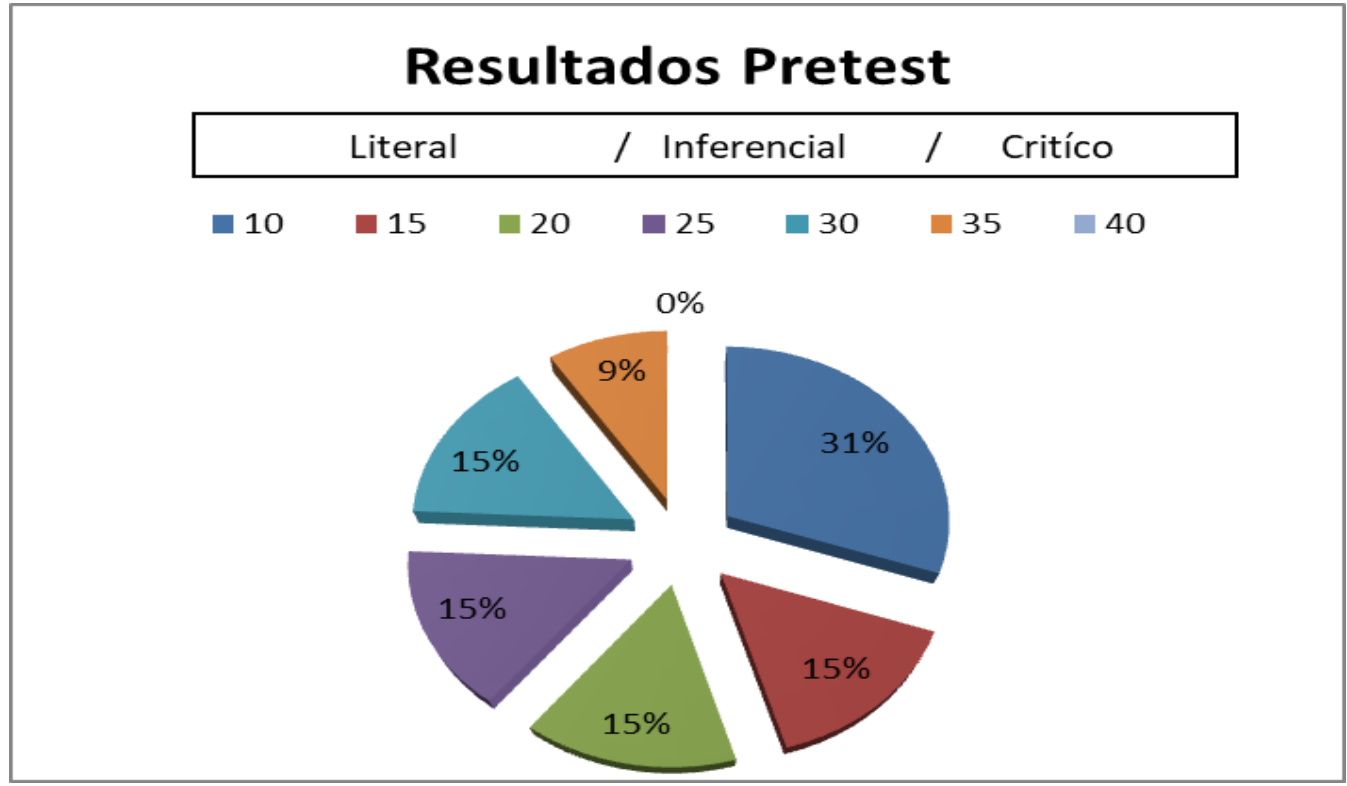

Fuente: Elaboración propia 


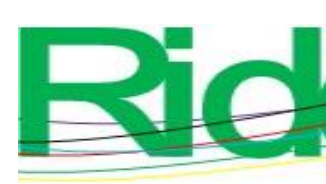

Revista Iberoamericana para la Investigación y el Desarrollo Educativo ISSN 2007 - 7467

Figura 2. Resultados postest

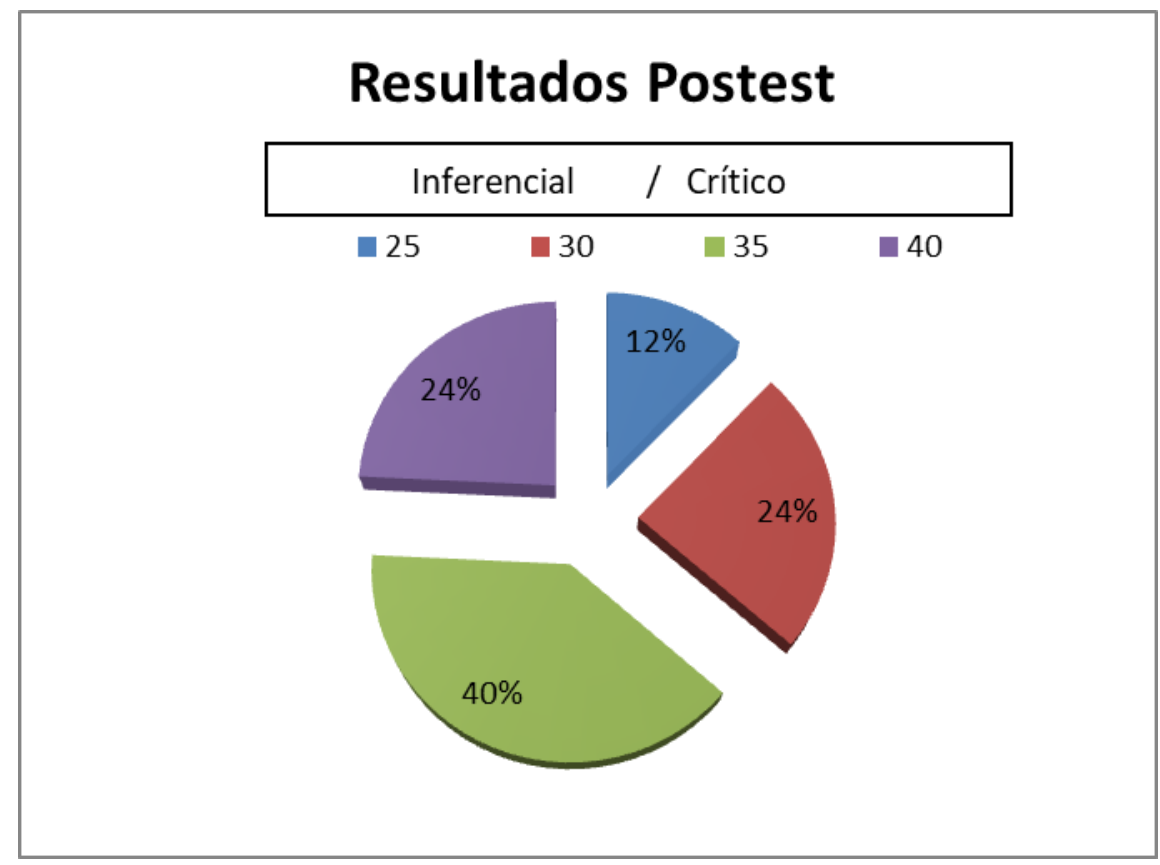

Fuente: Elaboración propia

\section{Discusión}

Las estrategias de lectura, según datos arrojados en el diagnóstico, no son utilizadas frecuentemente por los participantes de estudio. Lo anterior se resume de la siguiente manera: no usan el subrayado en $39 \%$ de los casos; $40 \%$ no identifica las ideas esenciales; los resúmenes y las síntesis, en un promedio de $84 \%$, no suelen ser empleados; $91 \%$ no elabora mapas conceptuales; $35 \%$ no acostumbra buscar el significado de las palabras que desconoce; $76 \%$ no maneja el planteamiento de hipótesis, y $46 \%$ no utiliza la reflexión como un proceso de análisis de la información. Estos resultados muestran que existe la necesidad de formar a los estudiantes, incluso en esta etapa de estudio, en el manejo de estrategias de apoyo a la lectura con la intención de favorecer la comprensión.

La medición de la comprensión lectora con la aplicación del diseño preexperimental con un solo grupo pretest y postest permitió la comprobación de la hipótesis. Se siguieron tres momentos según su procedimiento. Primero se aplicó el cuestionario (pretest) para evaluar la comprensión lectora, que situó a $45 \%$ de los estudiantes en el nivel inferencial, a $9 \%$ en el nivel crítico y a $46 \%$ en el nivel literal. El segundo paso consistió en la intervención educativa. Aquí se instruyó a los participantes de estudio en el uso de estrategias básicas de lectura, cuya 


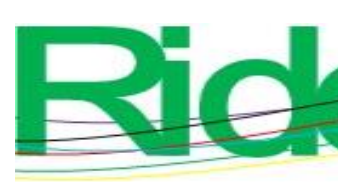

Revista Iberoamericana para la Investigación y el Desarrollo Educativo ISSN $2007-7467$

asistencia al taller en cada una de las cinco sesiones contó con más de $90 \%$ de asistencia. Y en el tercer paso se volvió a evaluar el nivel de comprensión lectora (postest), donde resultó que 64 \% alcanzó el nivel crítico y $36 \%$ se situó en el nivel inferencial; como queda evidente, nadie se posicionó en el nivel literal. Parte del análisis de los resultados fue calcular las medidas de tendencia central. Y en este paso se obtuvo que la media del puntaje de la evaluación pretest fue de 25 puntos, equivalente al nivel inferencial, y la media del puntaje de la evaluación postest llegó a los 35 puntos, correspondiente al nivel crítico, lo que demuestra la mejora del nivel de comprensión lectora de los alumnos involucrados.

Los datos obtenidos refieren que en la evaluación postest $38 \%$ de los participantes avanzaron de nivel; de estos, $2 \%$ adelantó dos niveles y $36 \%$ un nivel. En cuanto a puntaje, el resultado a favor fue más contundente. Aquí, $88 \%$ mejoró respecto a los resultados del pretest, puesto que, el progreso se observó con el incremento en puntos de la siguiente forma: $37 \%$ obtuvo cinco puntos más, $33 \% 10$ puntos más, $12 \% 15$ puntos más, $3 \% 20$ puntos más, $3 \% 25$ puntos más y $12 \%$ quedó en el mismo puntaje (de estos, $6 \%$ obtuvo el mayor puntaje en ambas evaluaciones). Cabe destacar que $50 \%$ de los casos, aunque no logró cambiar de nivel, mejoró su comprensión lectora al obtener mayor puntaje. Estos datos finales permitieron aceptar la hipótesis planteada.

Dentro de las limitaciones del estudio se encuentra que el diagnóstico fue con base en la opinión de los participantes de estudio, puesto que, al no contar con los tiempos y espacios suficientes, fue complicado poder comprobar cómo utilizan las estrategias de apoyo a la lectura, es decir, no se corrobora cuál es la realidad de su uso ni tampoco qué tan efectivamente las utilizan, lo cual pudiera ser tema de futuras investigaciones. Otra cuestión para tomar en cuenta es que solo se pudo trabajar con un grupo y no con los cuatro inscritos en primer semestre en el tiempo que se llevó a cabo la investigación. Lo anterior pudiera solucionarse al involucrar a más docentes en la instrucción y autoridades en la atención conjunta del problema. Asimismo, una línea interesante para otro trabajo investigativo pudiera ser la incidencia de la competencia lectora en el aprendizaje de los universitarios. 


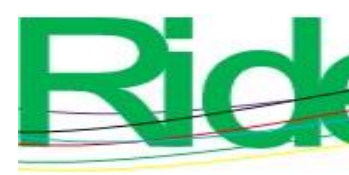

Revista Iberoamericana para la
Investigación y el Desarrollo Educativo
ISSN $2007-7467$

\section{Referencias}

Arias, F. (2012). El proyecto de investigación. Introducción a la metodología científica (6. ${ }^{\mathrm{a}}$ ed.). Caracas, Venezuela: Editorial Episteme. Recuperado de https://ebevidencia.com/wpcontent/uploads/2014/12/EL-PROYECTO-DE-INVESTIGACI\%C3\%93N-6ta-Ed.FIDIAS-G.-ARIAS.pdf

Buendía, L., Colás, P. y Hernández, F. (2000). Métodos de investigación psicopedagógica. Madrid, España: McGraw-Hill.

Cáceres, L., Pérez, C. y Zúñiga, M. (2018). Reflexiones teórico-metodológicas que sustentan el papel de la lectura y su comprensión en la renovación de los procesos de enseñanza y aprendizaje en el currículo universitario. Universidad y Sociedad, 10(4), 110-119.

Cassany, D. (2006). Tras las líneas. Sobre la lectura contemporánea. Barcelona, España: Anagrama. Recuperado de https://media.utp.edu.co/referenciasbibliograficas/uploads/referencias/libro/295-tras-las-lneaspdf-WB5V4-articulo.pdf

Corbacho, A. (2006). Textos, tipos de texto y textos especializados. Revista de Filología, (24), 77-90. Recuperado de https://dialnet.unirioja.es/servlet/articulo?codigo=2100070

Espinosa, A. (2016). La competencia lectora en la universidad: Una intervención didáctica como base de alfabetización académica. Colombia: Editorial Redipe.

Felipe, A. y Barrios, E. (2017). Evaluación de la competencia lectora de futuros docentes. Investigaciones sobre Lectura, (7), 7-21. Recuperado de https://dialnet.unirioja.es/servlet/articulo?codigo $=5891268$

Gaeta, M. (2015). Aspectos personales que favorecen la autorregulación del aprendizaje en la comprensión de textos académicos en estudiantes universitarios. REDU. Revista de la Docencia Universitaria, 13(2), 17-35. Recuperado de https://polipapers.upv.es/index.php/REDU/article/view/5436/5416

Gordillo, A. y Flores. M. (2009). Los niveles de comprensión lectora: hacia una enunciación investigativa y reflexiva para mejorar la comprensión lectora en estudiantes universitarios. Revista Actualidades Pedagógicas, (53), 95-107. Recuperado de https://ciencia.lasalle.edu.co/cgi/viewcontent.cgi?article=1100\&context=ap

Guevara, Y., Guerra, J., Delgado, U. y Flores, C. (2014). Evaluación de distintos niveles de comprensión lectora en estudiantes mexicanos de psicología. Acta Colombiana de Psicología, 17(2), 113-121. 


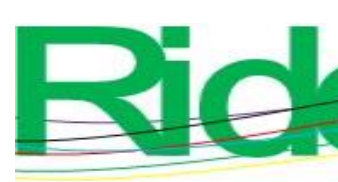

Revista Iberoamericana para la
Investigación y el Desarrollo Educativo
ISSN $2007-7467$

Gutiérrez, A. y Montes de Oca, R. (2004). La importancia de la lectura y su problemática en el contexto educativo universitario. El caso de la Universidad Juárez Autónoma de Tabasco, México. Revista Iberoamericana de Educación, 34(3). Recuperado de https://rieoei.org/RIE/article/view/3265.

Hernández, R. y Mendoza, C. (2018) Metodología de la investigación: Las rutas cuantitativa, cualitativa y mixta. Ciudad de México, México: McGraw-Hill. Recuperado de https://books.google.com.mx/books?hl=es\&lr=\&id=5A2QDwAAQBAJ\&oi=fnd\&pg=PP $1 \& \mathrm{dq}=$ investigaci\%C3\%B3n+cuantitativa+sampieri\&ots=TiYkYWZpL2\&sig=XXODO3 zCcdYm6D3oJ4XaNEEKDyM\#v=onepage\&q=investigaci\%C3\%B3n\%20cuantitativa\%2 Osampieri\&f=falsev.

Organización para la Cooperación y el Desarrollo Económicos [OCDE]. (2016). Programa para la Evaluación de Alumnos (PISA). PISA (2015) - Resultados. Nota País. Recuperado de https://www.oecd.org/pisa/PISA-2015-Mexico-ESP.pdf.

Organización para la Cooperación y el Desarrollo Económicos [OCDE]. (2007). El Programa PISA de la OCDE. Qué es y para qué sirve. París, Francia: Organización para la Cooperación y el Desarrollo Económicos. Recuperado de https://www.oecd.org/pisa/39730818.pdf

Osorio, V., Mendoza, E. y Ballesteros, E. (2018). La importancia de la lectura en el desarrollo de las habilidades investigativas del estudiante universitario. Revista Ciencias Sociales y Económicas, $\quad$ 2, 7-91. Recuperado de http://revistas.uteq.edu.ec/index.php/csye/article/view/219/165.

Parodi, G., Peronard, M. e Ibáñez, R. (2010). Saber leer. Madrid, España: Aguilar. Recuperado de https://issuu.com/alexrivera20/docs/saber_leer

Pérez, M. (2005). Evaluación de la comprensión lectora: dificultades y limitaciones. Revista de Educación, (núm. extraordinario), 121-138. Recuperado de http://www.oei.es/evaluacioneducativa/evaluacion_comprension_lectora_perez_zorrilla.p df.

Solé, I. (2011). Estrategias de lectura. España: GRAÓ

Van Dijk, T. \& Kintsch, W. (1983) Strategies of Discourse Comprehension. New York, United States: Academic Press. Retrieved from http://www.discourses.org/OldBooks/Teun\%20A\%20van\%20Dijk\%20\&\%20Walter\%20 Kintsch\%20-\%20Strategies\%20of\%20Discourse\%20Comprehension.pdf. 


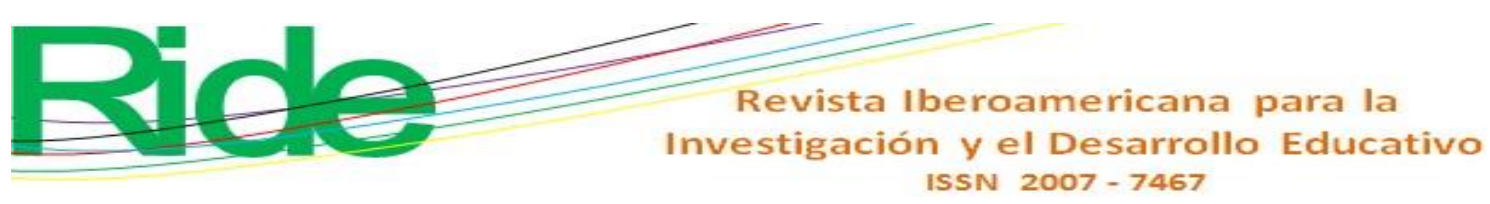

Vidal, D. y Manríquez, L. (2016). El docente como mediador de la comprensión lectora en universitarios. Revista de la Educación Superior, 45(177), 95-118. Recuperado de http://dx.doi.org/10.1016/j.resu.2016.01.009 